\title{
Why is it so difficult to evaluate faecal microbiota transplantation as a treatment for ulcerative colitis?
}

\author{
Natalie Grace Fairhurst ${ }^{1}$, Simon P. L. Travis ${ }^{2}$ \\ ${ }^{1}$ St Catherine's College, University of Oxford, Oxford, ${ }^{2}$ Translational Gastroenterology Unit, Nuffield Department of Experimental Medicine, \\ John Radcliffe Hospital, Oxford, UK
}

Faecal microbiota transplantation (FMT) has recently re-emerged as a viable therapeutic option for colonic disorders. Its efficacy has been proved in the treatment of Clostridium difficile infection which has encouraged research into the use of FMT for other disorders involving gut dysbiosis, such as ulcerative colitis (UC), a chronic inflammatory disease characterized by relapsing and remitting colonic inflammation. Although the FMT protocol for $C$. difficile treatment is well established, there are numerous additional factors to consider when applying FMT to treat inflammatory diseases. Various studies have attempted to address these factors but technical inconsistency between reports has resulted in a failure to achieve clinically significant findings. Case reports of FMT in UC have shown favorable outcomes yet demonstrating these effects on a larger scale has proved difficult. The following review aims to explore these issues and to analyze why they may be hindering the progression of FMT therapy in UC. (Intest Res 2018;16:209-215)

Key Words: Colitis, ulcerative; Fecal microbiota transplantation

\section{INTRODUCTION}

The gastrointestinal tract is estimated to host over $10^{14}$ bacteria. ${ }^{1}$ The composition of this colonic community has been linked to various aspects of human health. ${ }^{2-4}$ Manipulation of the gut flora offers insight into the underlying cause of a number of diseases and may, in some cases, be of therapeutic potential.

Current methods of altering the microbial composition include antibiotics, probiotics and prebiotics but evidence in support of their therapeutic benefit is limited. ${ }^{5,6}$ Recent attention has turned to the emerging method of microbial manipulation known as faecal microbiota transplantation (FMT). ${ }^{7}$ Broadly, FMT involves the transfer of a full spectrum of enteric microbiota from a healthy donor into a recipient's

Received August 20, 2017. Revised September 6, 2017.

Accepted September 6, 2017. Published online February 1, 2018

Correspondence to Simon P. L. Travis, Translational Gastroenterology Unit,

Nuffield Department of Experimental Medicine, University of Oxford, Oxford,

OX3 9DU, UK. Tel: +44-1865-227552, Fax: +44-1865-228763, E-mail:

simon.travis@ndm.ox.ac.uk intestine with the principal aim of restoring the disturbed colonic environment and consequently reversing disease. ${ }^{8}$

The principle of faecal microbiota transplant has existed since the 4th century. Traditional Chinese medicine describes the use of "yellow soup" to successfully treat patients with severe diarrhoea. ${ }^{9}$ The first modern faecal transplant conducted in humans was performed in 1958 by Eiseman et al. ${ }^{10}$ as a treatment for pseudomembranous colitis. However, with the burgeoning antibiotic boom of the 1960s, interest in FMT as a clinical therapy was suspended until the turn of the century.

FMT was first introduced into clinical practice in 2014 as a primary treatment for recurrent Clostridium difficile infection (CDI). The infection thrives after antibiotic treatment which suppresses the normal gut microbiota, allowing resistant $C$. difficile to germinate. The spore-forming bacterium produces enterotoxins which cause colonic inflammation and watery diarrhoea. ${ }^{11}$ Paradoxically, the primary treatment for $C$. difficile is antimicrobials. However, in $20 \%$ to $30 \%$ of patients, this is unsuccessful and leads to recurrent infection. $^{12}$

๑ Copyright 2018. Korean Association for the Study of Intestinal Diseases. All rights reserved.

This is an Open Access article distributed under the terms of the Creative Commons Attribution Non-Commercial License (http://creativecommons.org/licenses/by-nc/4.0)

which permits unrestricted non-commercial use, distribution, and reproduction in any medium, provided the original work is properly cited. 
Two major multicenter studies conducted by Bakken et al. ${ }^{13}$ and van Nood et al. ${ }^{14}$ both show successful use of faecal transplantation to treat $C$. difficile (up to $98 \%$ success rate compared with conventional vancomycin therapy at $31 \%$ ). This has fuelled interest in the use of FMT for a variety of other colonic conditions, including inflammatory diseases such as UC.

UC is a chronic inflammatory disease affecting the distal colon and rectum which predominantly presents in young adults. ${ }^{15}$ Its aetiology is unknown but it is thought to result from an abnormal immune response to alteration in the gut environment in genetically susceptible individuals. The symptoms of active disease include bloody diarrhoea and abdominal pain. ${ }^{16}$ Current therapy is primarily targeted at the aberrant immune response but achieves limited success. ${ }^{17}$ Instead, focus has turned to the dysbiosis that is thought to contribute to UC and thus FMT has emerged as a potential therapeutic option.

Recent publications have reported the effective treatment of UC with FMT but there are numerous studies showing conflicting results. ${ }^{18}$ With strong evidence supporting the use of FMT in disorders involving microbiota shifts, one must pose the question: why is it so difficult to evaluate FMT as a therapeutic option in UC?

Although there are currently 16 randomized control trials (RCTs) registered exploring the relationship between FMT and $\mathrm{UC},{ }^{19}$ most of the data thus far is based on case reports and small-scale studies. This is partly due to the novelty of the therapy in this field but is also because of the extensive time and financial support required for rigorous testing. Amongst the published data are 3 recent RCTs comparing the effectiveness of FMT against placebo.

Moayyedi et al. ${ }^{20}$ recruited 75 patients with active UC, assigning half to receive weekly FMT and the other half to receive water enema. The trial concluded a significant difference: $24 \%$ of the experiment group were in remission after 7 weeks compared with $5 \%$ of the placebo group. An opposing study by Rossen et al. ${ }^{21}$ was published within the same week. This trial observed 48 patients with active UC, giving 23 patients FMT and 25 patients an autologous faecal transplant twice-at week 0 and week 3 . Results showed no statistical significance between the 2 groups after 12 weeks (30\% remission in FMT group vs. 20\% in control). Given the similarity between these studies, one must wonder why there is such disparity in results. Subgroup analysis of both studies elucidated various issues. Moayyedi et al. ${ }^{20}$ note that there were donor dependent differences and possible "critical windows" of application whereas Rossen et al. ${ }^{21}$ suggest route of administration, dosing frequency and donor matching may account for these discrepancies.

More recent studies have aimed to address several of the shortcomings of these 2 conflicting RCTs. Paramsothy et al. ${ }^{22}$ recently conducted the largest multicenter RCT to date, aiming to identify the clinical benefit of FMT for UC in a study of 81 patients with active UC. By analyzing previous issues, the group was able to design a study that eliminated various factors. The trial concluded significant results with $27 \%$ of FMTreceivers reaching primary outcome (steroid-free clinical remission and endoscopic remission) after 8 weeks, versus 8\% with placebo $(P=0.02)$. In addition, a proportion of those who originally received placebo progressed to open-label FMT, with a further $27 \%$ achieving the primary end-point.

Although all 3 trials had analogous primary aims (to assess the efficacy of FMT in inducing remission of chronic UC), there remains significant disparity between study outcomes. Before FMT is to be considered as a possible therapeutic option, the cause for conflicting conclusions must be identified.

\section{PROFILING THE MICROBIOME}

Although FMT has proved successful in CDI, the dysbiosis associated with UC is far less pronounced than in CDI. Therefore, it is harder to identify specific microbial perturbations that may influence UC pathogenesis. In addition, every individual microbiome has a distinct microbial composition which makes homogenous FMT more difficult.

In order to map changes in microbial composition, most studies conduct microbiome profiling of both donors and patients. These studies have shown that diseased intestines often express a lower diversity of bacteria. ${ }^{23-25}$ The significance of this decreased diversity is that the functional composition of the microbiota is affected. Commensal bacteria normally provide protection against inflammatory diseases via colonization resistance and shaping the mucosal immune system. It is therefore not surprising that microbial disorder is a hallmark of UC.

Further profiling of the microbiota has elucidated differences in specific bacteria between test groups. The broad pattern of dysbiosis in inflammatory diseases is characterized by a reduction in Bacteroidetes and Firmicutes phylum and an increase in Proteobacteria. ${ }^{26-28}$ Moayyedi et al. ${ }^{20}$, Rossen et al. ${ }^{21}$ and Paramsothy et al., ${ }^{22}$ amongst other studies, show a significantly increased microbial diversity after treatment with FMT. Members of the Lachnospiraceae family and Ruminococcus genus are both notably depleted in UC and successful transplants have shown enrichment of these 


\section{2 bacteria. $^{20}$}

Most studies note a difference in the gut bacterial species in UC compared with healthy controls. However, it is unclear whether specific bacterial strains are responsible for disease induction or maintenance. One theory proposes that the immune dysregulation is driven by a change in the gut flora. However, others theorise that incidence of UC is significantly elevated in patients following an episode of acute gastroenteritis $^{29,30}$ because this infection leads to chronic changes in intestinal milieu allowing invasive bacteria to cause intestinal inflammation. Fluorescent in situ hybridization identifies these bacteria in $83 \%$ of tissue samples from UC sufferers compared to none in controls. ${ }^{31}$ If this latter theory is true, then FMT may be a futile therapy as the intestinal lining is irrevocably weakened and effects will merely be temporary.

Emerging data also implicates the microbiome in the pathology of various other non-intestinal diseases including mental health disorders, obesity and immune function. ${ }^{4,32,33}$ Whilst FMT has thus far shown few side effects, long-term intervention could potentially cause more harm than good if these side effects are not mitigated.

These issues highlight the need for careful donor selection and microbiome profiling (of both donor and recipient, before and after treatment). By monitoring the altered profile in disease, the luminal flora may predict response to therapy and indicate suitable patients who would benefit from FMT.

\section{THE PERFECT DONOR}

Although there may be key bacteria that could maximize treatment success, the current "one-stool-fits-all" approach may not be clinically appropriate. A number of studies have proposed the idea of a "super-donor." The concept of the super-donor originated in the study by Moayeddi et al. ${ }^{20}$ which showed statistical significance for donor dependence (of 9 patients who went into remission, 7 were from 1 donor). Taxonomic profile analysis revealed that donors displaying a particular microbial enrichment had a more efficacious stool.

Conversely, Paramsothy et al. ${ }^{22}$ adopted a multi-donor approach where each individual recipient was given a blended stool from between 3 and 7 donors. This method minimized the chance of a patient receiving only ineffectual FMT. Although this may reduce the ability to identify specific "superdonors" and their successful strains of bacteria, the richness of infusion and microbial heterogeneity may contribute to improved outcomes.

Moreover, pre and post-FMT microbiota analyses are lim- ited to species level comparisons and have not investigated the importance of donor-host strain differences. Through metagenomic analysis of single-nucleotide variants, one study was able to show that allogeneic recipients from a single donor show very significant single-nucleotide variants differences across specific bacterial populations. Furthermore, they showed that donor strains are able to cohabit with pre-existing isotypes. This suggests that the recipient has reciprocal effects on the sample, and that what may seem like a solely donor-dependent occurrence is actually a donor-recipient symbiosis. ${ }^{34}$

Patient-donor matching is of particular importance in paediatric FMT. Ribosomal RNA analysis of bacterial components in healthy children has identified "age-discriminatory" taxa whose abundance changes with normal maturation. ${ }^{35}$ This suggests that age-matched donors may be necessary for paediatric cases so that transplants reflect an equivalent immunological age.

In some cases, despite a "super-donor" transplant, recipients regress to the pre-FMT state indicating that donorrecipient compatibilities are crucial for FMT efficacy. Evidence suggests that there is no perfect donor for a successful FMT treatment and that the composition of both donor and recipient gut flora can influence efficacy. In some ways, this is not too different from typical organ transplant rejectionperhaps a more thorough selection method similar to major histocompatibility complex matching is necessary.

\section{CALL FOR A STANDARDISED PROTOCOL}

Part of the issue in obtaining significant results is the lack of standardized therapeutic protocol. Published case study series give conflicting results for FMT efficacy which could be at least partially attributable to the absence of congruent procedure. Trials conducted by Rossen et al. ${ }^{21}$ exemplify the importance of consistent preparation methods. Their study showed that even autologous FMT produces a change in the colonic microbiome suggesting that processing alone is able to alter sample composition. Although RCTs are more rigorous in their experimental approach, there are still technical inconsistencies to address.

\section{Preparation of Faecal Material}

Preparation of stool sample varies across studies. Prior to administration, the stool sample is homogenized, typically with water but other diluents such as milk and yogurt have been used. Samples are prepared in an aerobic environment 
and if not done quickly, the exposure to non-biological conditions could affect the microbial composition by favoring aerobes and killing anaerobes. Given that most of the gut bacteria are strict anaerobes, it may be beneficial to prepare to samples under anaerobic conditions. ${ }^{36}$

Additionally, some studies use fresh faecal material whereas others use frozen-thawed samples. Although the latter is easier for both patient and clinician, some suggest that the storage temperatures $\left(-20^{\circ}\right.$ to $\left.-80^{\circ}\right)$ can interfere with DNA. Comparisons of fresh versus frozen samples in $C$. difficile treatment have been conducted and conclude that there is no significant difference in efficacy. ${ }^{37}$ However, inflammatory diseases have a more complex pathology hence these results cannot be extrapolated to UC. A replicate comparison study of fresh versus frozen samples in UC would be beneficial. If frozen samples were found to be as efficacious as fresh samples, the logistics of treatment delivery would be simpler. One of the drawbacks of transplanting live bacteria between patients is the potential transfer of disease-causing pathogens. Although various groups show that FMT is safe even in immunocompromised patients, potential transmission of disease is still an underlying risk.

A preliminary investigation by Ott et al. ${ }^{38}$ addresses this issue by using faecal filtrates containing disassembled bacteria rather than intact organisms, supposedly reducing that chance of infectious transfer. In 5 patients with recurrent CDI given sterile faecal filtrate transfer via nasojejunal administration, symptoms were relieved after 3 days (on average), and patients remained symptom-free after 6 months. This was accompanied by a shift in microbial taxa. This potentially identifies a safer technique for transfer of the effective organism but whether this approach is transferable to inflammatory diseases demands further research.

\section{Preparation of the Patient}

In addition to preparation of the sample, some studies choose to "prepare" the recipient's colon with bowel lavage or a course of antibiotics whereas other groups opt for a nocleanse method. ${ }^{21}$ It may seem logical to clear the defective host bacteria before introduction of new flora but studies have shown that even infusion of polyethylene glycol (a bowel irrigation method) stimulates a shift in Proteobacteria colonization in healthy individuals. ${ }^{39}$ Pre-treatment of the recipient may produce results that are not attributable to FMT alone.

However, combination therapy alongside FMT may offer improved results. The study by Moayeddi et al. ${ }^{20}$ endorsed immunosuppressant therapy in conjunction with FMT. Subjects under immunosuppression achieved better outcomes (5 of 11 [46\%] in remission) that those solely on FMT (4 of 27 [15\%] in remission). Paramsothy et al. ${ }^{22}$ required that all participants be weaned from steroid treatment prior to the study commencing. Although this resulted in a number of individuals withdrawing, this method helps to isolate the efficacy of FMT alone.

\section{Timing of FMT}

There are many things to consider when choosing the best time to introduce FMT to the colon. Moayeddi et al. ${ }^{20}$ note a better outcome in newly diagnosed UC sufferers which suggests the presence of a "critical window" for administration. In early disease, the microbial perturbations may be more susceptible to reversal.

FMT success may also be contingent on the number of infusions given. There are drastic differences in frequency and duration of treatment ranging from single infusions to rigorous 40-infusion procedures. Some studies observe remission after a single transplant but lack of follow-up precludes conclusions about long-term effects. ${ }^{40}$

Paramsothy et al. ${ }^{22}$ followed 63 members of the effective cohort for a further 8 weeks after conclusion of the trial. Of this group, 23 remained in remission but 20 required additional treatment suggesting that FMT may be a chronic therapy rather than a "one-off" treatment. Continued intermittent FMT may be required to sustain UC remission and future studies should address this matter.

It may be that there is a FMT dose-response or threshold requirement in order to effectively reverse the inflammation in UC. Furthermore, rectification of dysbiosis in inflammatory diseases may only be transient. Patients could require chronic FMT therapy rather than a "one-off” treatment.

\section{Experimental Design}

One simple intervention to minimize experimental discrepancy is the use of a uniform disease scoring system. Although all 3 RCTs use the Mayo endoscopic scoring system, the primary end-points vary: Rossen et al. ${ }^{21}$ achieve Mayo score $\leq 1$ and simple clinical colitis index $\leq 2$ whereas Moayeddi et al. ${ }^{20}$ achieve endoscopic Mayo score $=0$ and overall score $<3$. Of the 3 RCTs, Paramsothy et al. ${ }^{22}$ demand the most stringent criterion for remission (that patients must show steroid-free clinical and endoscopic remission, with a Mayo score $\leq 2$ ) which adds weight to the study's design. 
If studies are achieving a different end-point, the number of subjects considered to "be in remission" is affected and cross-study conclusions cannot be drawn. ${ }^{41}$

The choice of placebo can also have significant effects on results. Moayeddi et al. ${ }^{20}$ opt for water enema with Paramsothy et al. ${ }^{22}$ electing isotonic saline (with brown coloring and odor to enhance double blinding), whereas Rossen et al. ${ }^{21}$ chose autologous faecal transplant. As previously mentioned, autologous transplants have been shown to affect the microbiota due to disturbances in aerobic/anaerobic bacteria which may give false-positive readings under placebo. Uniformity across studies would reduce unnecessary errors.

\section{Route of Administration}

Perhaps the most significant variation between the 3 studies is the route of administration. Moayeddi et al. ${ }^{20}$ and Paramsothy et al. ${ }^{22}$ introduce FMT via enema whereas Rossen et al. ${ }^{21}$ chose the nasoduodenal route. UC is primarily a disease of the colorectum and therefore delivery of FMT via the upper gastrointestinal tract may neutralise the constituents before arrival at the desired site. Equally, in extended UC, rectal enema may be insufficient to treat the whole colon. If certain bacteria are to be introduced, multiple routes of administration may be required: Firmicutes are $\mathrm{pH}$ tolerant and can survive the upper gastrointestinal tract whereas Bacteroidetes are susceptible to gastric acid. ${ }^{42}$ The optimal administration route will depend upon discovery of the key therapeutic bacteria.

Of course, each individual microbiota has a different composition which may partially account for the heterogeneity of the results: each FMT leads to donor-dependent effects. Therefore, in order to correctly attribute changes to FMT alone (and not to experimental fluctuations), future randomized trials must adopt a consistent protocol.

Despite the obvious differences in experimental design between these studies, the major determining factor producing the variation in results remains unidentified. Further research to isolate each of these potential contributory influences would be of benefit if FMT is to appear alongside current treatment for UC and other inflammatory diseases.

These studies show that FMT may provide a viable option for treating UC in future. However, one of the crucial considerations that has not been addressed in any study is the long-term effect of FMT. Studies in mice show that the gut microbial composition can alter susceptibility to disease alongside recent data which link microbial disturbances to mental health problems. ${ }^{33}$ Paradoxically, curing one disease by correcting microbial disturbances may trigger development of another. If FMT is to progress as a therapeutic option, these long-term consequences must be considered.

\section{CONCLUSIONS}

The major limitation to both Rossen et al.'s ${ }^{21}$ and Moayeddi et al.' $\mathrm{s}^{20}$ studies is that they overestimated the success of FMT. As a consequence, both studies were prematurely terminated. However, retrospective analysis of their combined results shows that the trials are in fact concurrent and support FMT as a favorable treatment when compared to current therapies.

Despite these positive results, it seems faecal microbiota transplant is not yet ready to replace existing treatment for treating UC in future. Although the most recent study by Paramsothy et al. ${ }^{22}$ addresses many of the previously raised issues, there are still aspects of both FMT and UC yet to be discovered. The unidentified aetiology of UC hinders therapeutic progression in this field but whilst the definitive cause is being sought, FMT could be offered as a complementary treatment in conjunction with existing therapies.

\section{FINANCIAL SUPPORT}

The authors received no financial support for the research, authorship, and/or publication of this article.

\section{CONFLICT OF INTEREST}

No potential conflict of interest relevant to this article was reported.

\section{AUTHOR CONTRIBUTION}

Natalie Grace Fairhurst: author, data review and analysis Simon P.L. Travis: revision of manuscript

\section{REFERENCES}

1. Savage DC. Microbial ecology of the gastrointestinal tract. Annu Rev Microbiol 1977;31:107-133.

2. Cryan JF, Dinan TG. Mind-altering microorganisms: the impact of the gut microbiota on brain and behaviour. Nat Rev Neurosci 2012;13:701-712.

3. Belkaid Y, Hand TW. Role of the microbiota in immunity and inflammation. Cell 2014;157:121-141. 
4. Turnbaugh PJ, Ley RE, Mahowald MA, Magrini V, Mardis ER, Gordon JI. An obesity-associated gut microbiome with increased capacity for energy harvest. Nature 2006;444:10271031.

5. Sartor RB. Therapeutic manipulation of the enteric microflora in inflammatory bowel diseases: antibiotics, probiotics, and prebiotics. Gastroenterology 2004;126:1620-1633.

6. Preidis GA, Versalovic J. Targeting the human microbiome with antibiotics, probiotics, and prebiotics: gastroenterology enters the metagenomics era. Gastroenterology 2009;136:2015-2031.

7. Cammarota G, Ianiro G, Tilg H, et al. European consensus conference on faecal microbiota transplantation in clinical practice. Gut 2017;66:569-580.

8. Khoruts A, Sadowsky MJ. Understanding the mechanisms of faecal microbiota transplantation. Nat Rev Gastroenterol Hepatol 2016;13:508-516.

9. Ge H. Zhou Hou Bei Ji Fang. Tianjin: Tianjin Science \& Technology Press, 2000

10. Eiseman B, Silen W, Bascom GS, Kauvar AJ. Fecal enema as an adjunct in the treatment of pseudomembranous enterocolitis. Surgery 1958;44:854-859.

11. Kelly CP, LaMont JT. Clostridium difficile infection. Annu Rev Med 1998;49:375-390.

12. Depestel DD, Aronoff DM. Epidemiology of Clostridium difficile infection. J Pharm Pract 2013;26:464-475.

13. Bakken JS, Borody T, Brandt LJ, et al. Treating Clostridium difficile infection with fecal microbiota transplantation. Clin Gastroenterol Hepatol 2011;9:1044-1049.

14. van Nood E, Vrieze A, Nieuwdorp M, et al. Duodenal infusion of donor feces for recurrent Clostridium difficile. N Engl J Med 2013;368:407-415.

15. Ford AC, Moayyedi P, Hanauer SB. Ulcerative colitis. BMJ 2013;346:f432. doi: 10.1136/bmj.f432.

16. Zhang YZ, Li YY. Inflammatory bowel disease: pathogenesis. World J Gastroenterol 2014;20:91-99.

17. Peyrin-Biroulet L, Lémann M. Review article: remission rates achievable by current therapies for inflammatory bowel disease. Aliment Pharmacol Ther 2011;33:870-879.

18. Paramsothy S, Paramsothy R, Rubin DT, et al. Faecal microbiota transplantation for inflammatory bowel disease: a systematic review and meta-analysis. J Crohns Colitis 2017;11:1180-1199.

19. Search UC + FMT. U.S. National Library of Medicine ClinicalTrials.gov. https://clinicaltrials.gov. Accessed April 10, 2017.

20. Moayyedi P, Surette MG, Kim PT, et al. Fecal microbiota transplantation induces remission in patients with active ulcerative colitis in a randomized controlled trial. Gastroenterology 2015;149:102-109.e6.
21. Rossen NG, Fuentes S, van der Spek MJ, et al. Findings from a randomized controlled trial of fecal transplantation for patients with ulcerative colitis. Gastroenterology 2015;149:110-118.e4.

22. Paramsothy S, Kamm MA, Kaakoush NO, et al. Multidonor intensive faecal microbiota transplantation for active ulcerative colitis: a randomised placebo-controlled trial. Lancet 2017;389:1218-1228.

23. Frank DN, St Amand AL, Feldman RA, Boedeker EC, Harpaz N, Pace NR. Molecular-phylogenetic characterization of microbial community imbalances in human inflammatory bowel diseases. Proc Natl Acad Sci U S A 2007;104:13780-13785.

24. Ott SJ, Musfeldt M, Wenderoth DF, et al. Reduction in diversity of the colonic mucosa associated bacterial microflora in patients with active inflammatory bowel disease. Gut 2004;53:685693.

25. Sha S, Xu B, Wang X, et al. The biodiversity and composition of the dominant fecal microbiota in patients with inflammatory bowel disease. Diagn Microbiol Infect Dis 2013;75:245-251.

26. Peterson DA, Frank DN, Pace NR, Gordon JI. Metagenomic approaches for defining the pathogenesis of inflammatory bowel diseases. Cell Host Microbe 2008;3:417-427.

27. Manichanh C, Rigottier-Gois L, Bonnaud E, et al. Reduced diversity of faecal microbiota in Crohn's disease revealed by a metagenomic approach. Gut 2006;55:205-211.

28. Gophna U, Sommerfeld K, Gophna S, Doolittle WF, Veldhuyzen van Zanten SJ. Differences between tissue-associated intestinal microfloras of patients with Crohn's disease and ulcerative colitis. J Clin Microbiol 2006;44:4136-4141.

29. Molodecky NA, Kaplan GG. Environmental risk factors for inflammatory bowel disease. Gastroenterol Hepatol (N Y) 2010;6:339-346.

30. García Rodríguez LA, Ruigómez A, Panés J. Acute gastroenteritis is followed by an increased risk of inflammatory bowel disease. Gastroenterology 2006;130:1588-1594.

31. Sasaki M, Klapproth JM. The role of bacteria in the pathogenesis of ulcerative colitis. J Signal Transduct 2012;2012:704953. doi: 10.1155/2012/704953.

32. Round JL, Mazmanian SK. The gut microbiota shapes intestinal immune responses during health and disease. Nat Rev Immunol 2009;9:313-323.

33. Bercik P, Denou E, Collins J, et al. The intestinal microbiota affect central levels of brain-derived neurotropic factor and behavior in mice. Gastroenterology 2011;141:599-609.

34. Li SS, Zhu A, Benes V, et al. Durable coexistence of donor and recipient strains after fecal microbiota transplantation. Science 2016;352:586-589. 
35. Subramanian S, Huq S, Yatsunenko T, et al. Persistent gut microbiota immaturity in malnourished Bangladeshi children. Nature 2014;510:417-421.

36. Gough E, Shaikh H, Manges AR. Systematic review of intestinal microbiota transplantation (fecal bacteriotherapy) for recurrent Clostridium difficile infection. Clin Infect Dis 2011;53:9941002.

37. Lee CH, Steiner T, Petrof EO, et al. Frozen vs fresh fecal microbiota transplantation and clinical resolution of diarrhea in patients with recurrent Clostridium difficile infection: a randomized clinical trial. JAMA 2016;315:142-149.

38. Ott SJ, Waetzig GH, Rehman A, et al. Efficacy of sterile fecal filtrate transfer for treating patients with Clostridium difficile infection. Gastroenterology. 2017;152:799-811.e7.
39. Jalanka J, Salonen A, Salojärvi J, et al. Effects of bowel cleansing on the intestinal microbiota. Gut 2015;64:1562-1568.

40. Cui B, Feng Q, Wang H, et al. Fecal microbiota transplantation through mid-gut for refractory Crohn's disease: safety, feasibility, and efficacy trial results. J Gastroenterol Hepatol 2015;30:5158.

41. Walsh AJ, Bryant RV, Travis SP. Current best practice for disease activity assessment in IBD. Nat Rev Gastroenterol Hepatol 2016;13:567-579.

42. Flint HJ, Scott KP, Louis P, Duncan SH. The role of the gut microbiota in nutrition and health. Nat Rev Gastroenterol Hepatol 2012;9:577-589 